1996

\title{
Cardinal Invariants Concerning Extendable and Peripherally Continuous Functions
}

Krzysztof Ciesielski

West Virginia University, krzysztof.ciesielski@mail.wvu.edu

Follow this and additional works at: https://researchrepository.wvu.edu/faculty_publications

Part of the Mathematics Commons

\section{Digital Commons Citation}

Ciesielski, Krzysztof, "Cardinal Invariants Concerning Extendable and Peripherally Continuous Functions" (1996). Faculty Scholarship. 823.

https://researchrepository.wvu.edu/faculty_publications/823 
Krzysztof Ciesielski, Department of Mathematics, West Virginia University, Morgantown, WV 26506-6310, e-mail: kcies@@wvnvms.wvnet.edu Ireneusz Recław*, Department of Mathematics, University of Scranton, Scranton, PA 18510-4666, e-mail: ReclawI1@@jaguar.uofs.edu permanent address: Institute of Mathematics, Gdańsk University, Wita Stwosza 57, 80-952 Gdańsk, Poland, e-mail: matir@@halina.univ.gda.pl

\title{
CARDINAL INVARIANTS CONCERNING EXTENDABLE AND PERIPHERALLY CONTINUOUS FUNCTIONS
}

\begin{abstract}
Let $\mathcal{F}$ be a family of real functions, $\mathcal{F} \subseteq \mathbb{R}^{\mathbb{R}}$. In the paper we will examine the following question. For which families $F \subseteq \mathbb{R}^{\mathbb{R}}$ does there exist $g: \mathbb{R} \rightarrow \mathbb{R}$ such that $f+g \in \mathcal{F}$ for all $f \in F$ ? More precisely, we will study a cardinal function $\mathrm{A}(\mathcal{F})$ defined as the smallest cardinality of a family $F \subseteq \mathbb{R}^{\mathbb{R}}$ for which there is no such $g$. We will prove that $\mathrm{A}($ Ext $)=\mathrm{A}(\mathrm{PR})=\mathfrak{c}^{+}$and $\mathrm{A}(\mathrm{PC})=2^{\mathfrak{c}}$, where Ext, PR and $\mathrm{PC}$ stand for the classes of extendable functions, functions with perfect road and peripherally continuous functions from $\mathbb{R}$ into $\mathbb{R}$, respectively. In particular, the equation $\mathrm{A}(\mathrm{Ext})=\mathfrak{c}^{+}$immediately implies that every real function is a sum of two extendable functions. This solves a problem of Gibson [6].

We will also study the multiplicative analogue $\mathrm{M}(\mathcal{F})$ of the function $\mathrm{A}(\mathcal{F})$ and we prove that $\mathrm{M}($ Ext $)=\mathrm{M}(\mathrm{PR})=2$ and $\mathrm{A}(\mathrm{PC})=\mathfrak{c}$.

This article is a continuation of papers $[10,3,12]$ in which functions $\mathrm{A}(\mathcal{F})$ and $\mathrm{M}(\mathcal{F})$ has been studied for the classes of almost continuous, connectivity and Darboux functions.
\end{abstract}

\footnotetext{
Key Words: cardinal invariants, extendable functions, functions with prefect road, peripherally continuous functions

Mathematical Reviews subject classification: Primary: 26A15. Secondary: 03E75, $54 \mathrm{~A} 25$

Received by the editors October 26, 1994

*The article was written when the second author was working at Institut für Mathematik II, FU Berlin, Arnimallee 3, 14195 Berlin, Germany, under the support of Alexander von Humboldt Foundation
} 


\section{Introduction}

We will use the following terminology and notation. Functions will be identified with their graphs. The family of all functions from a set $X$ into $Y$ will be denoted by $Y^{X}$. The symbol $|X|$ will stand for the cardinality of a set $X$. The cardinality of the set $\mathbb{R}$ of real numbers is denoted by $\mathfrak{c}$. For a cardinal number $\kappa$ we will write $\operatorname{cf}(\kappa)$ for the cofinality of $\kappa$. A cardinal number $\kappa$ is regular, if $\kappa=\operatorname{cf}(\kappa)$. For $A \subseteq \mathbb{R}$ its characteristic function is denoted by $\chi_{A}$. In particular, $\chi_{\emptyset}$ stands for the zero constant function.

In his study of the class D of Darboux functions (See definition below.) Fast [5] proved that for every family $F \subseteq \mathbb{R}^{\mathbb{R}}$ of cardinality at most that of the continuum there exists $g: \mathbb{R} \rightarrow \mathbb{R}$ such that $f+g$ is Darboux for every $f \in F$. Natkaniec [10] proved the similar result for the class AC of almost continuous functions and defined the following two cardinal invariants for every $\mathcal{F} \subseteq \mathbb{R}^{\mathbb{R}}$.

$$
\begin{aligned}
\mathrm{A}(\mathcal{F}) & =\min \left\{|F|: F \subseteq \mathbb{R}^{\mathbb{R}} \& \neg \exists g \in \mathbb{R}^{\mathbb{R}} \forall f \in F f+g \in \mathcal{F}\right\} \cup\left\{\left(2^{\mathfrak{c}}\right)^{+}\right\} \\
& =\min \left\{|F|: F \subseteq \mathbb{R}^{\mathbb{R}} \& \forall g \in \mathbb{R}^{\mathbb{R}} \exists f \in F f+g \notin \mathcal{F}\right\} \cup\left\{\left(2^{\mathfrak{c}}\right)^{+}\right\}
\end{aligned}
$$

and

$$
\begin{aligned}
\mathrm{M}(\mathcal{F}) & =\min \left\{|F|: F \subseteq \mathbb{R}^{\mathbb{R}} \& \neg \exists g \in \mathbb{R}^{\mathbb{R}} \backslash\left\{\chi_{\emptyset}\right\} \forall f \in F f \cdot g \in \mathcal{F}\right\} \cup\left\{\left(2^{\mathfrak{c}}\right)^{+}\right\} \\
& =\min \left\{|F|: F \subseteq \mathbb{R}^{\mathbb{R}} \& \forall g \in \mathbb{R}^{\mathbb{R}} \backslash\left\{\chi_{\emptyset}\right\} \exists f \in F f \cdot g \notin \mathcal{F}\right\} \cup\left\{\left(2^{\mathfrak{c}}\right)^{+}\right\} .
\end{aligned}
$$

Thus, Fast and Natkaniec effectively showed that $A(D)>\mathfrak{c}$ and $A(A C)>\mathfrak{c}$.

The extra assumption that $g \neq \chi_{\emptyset}$ is added in the definition of $\mathrm{M}$ since otherwise for every family $\mathcal{F} \subseteq \mathbb{R}^{\mathbb{R}}$ containing $\chi_{\emptyset}$ we would have $\mathrm{M}(\mathcal{F})=\left(2^{\mathfrak{c}}\right)^{+}$.

Notice the following basic properties of functions A and M.

Proposition 1.1 Let $\mathcal{F} \subseteq \mathcal{G} \subseteq \mathbb{R}^{\mathbb{R}}$.

(1) $\mathrm{A}(\mathcal{F}) \leq \mathrm{A}(\mathcal{G})$.

(2) $\mathrm{A}(\mathcal{F}) \geq 2$ if $\mathcal{F} \neq \emptyset$.

(3) $\mathrm{A}(\mathcal{F}) \leq 2^{\mathfrak{c}}$ if $\mathcal{F} \neq \mathbb{R}^{\mathbb{R}}$.

Proof. (1) is obvious. To see (2) let $h \in \mathcal{F}$ and $F=\{f\}$ for some $f \in \mathbb{R}^{\mathbb{R}}$. Then $f+g \in \mathcal{F}$ for $g=h-f$. To see (3) note that for $F=\mathbb{R}^{\mathbb{R}}$ and every $g \in \mathbb{R}^{\mathbb{R}}$ there is $f \in F$ with $f+g \notin \mathcal{F}$, namely $f=h-g$, where $h \in \mathbb{R}^{\mathbb{R}} \backslash \mathcal{F}$.

Proposition 1.2 Let $\mathcal{F} \subseteq \mathcal{G} \subseteq \mathbb{R}^{\mathbb{R}}$.

(1) $\mathrm{M}(\mathcal{F}) \leq \mathrm{M}(\mathcal{G})$.

(2) $\mathrm{M}(\mathcal{F}) \geq 2$ if $\chi_{\emptyset}, \chi_{\mathbb{R}} \in \mathcal{F}$.

(3) $\mathrm{M}(\mathcal{F}) \leq \mathfrak{c}$ if $r \chi_{\{x\}} \notin \mathcal{F}$ for every $r, x \in \mathbb{R}, r \neq 0$. 
Proof. (1) is obvious. To see (2) let $F=\{f\}$ for some $f \in \mathbb{R}^{\mathbb{R}}$. If there is $x \in \mathbb{R}$ such that $f(x)=0$ take $g=\chi_{\{x\}}$. Otherwise, define $g(x)=1 / f(x)$ for every $x \in \mathbb{R}$. Then $f \cdot g \in\left\{\chi_{\emptyset}, \chi_{\mathbb{R}}\right\} \subseteq \mathcal{F}$. To see (3) note that for $F=\left\{\chi_{\{x\}}: x \in \mathbb{R}\right\}$ and every $g \in \mathbb{R}^{\mathbb{R}} \backslash\left\{\chi_{\emptyset}\right\}$ there is $f \in F$ with $f \cdot g \notin \mathcal{F}$, namely $f=\chi_{\{x\}}$, where $x$ is such that $g(x)=r \neq 0$.

Proposition 1.3 Let $\chi_{\emptyset} \in \mathcal{F} \subseteq \mathbb{R}^{\mathbb{R}}$. Then $\mathrm{A}(\mathcal{F})=2$ if and only if $\mathcal{F}-\mathcal{F}=$ $\left\{f_{1}-f_{2}: f_{1}, f_{2} \in \mathcal{F}\right\} \neq \mathbb{R}^{\mathbb{R}}$.

Proof. " $\Rightarrow$ " Assume that $\mathcal{F}-\mathcal{F}=\mathbb{R}^{\mathbb{R}}$. We will show that $\mathrm{A}(\mathcal{F})>2$. So, pick arbitrary $f_{1}, f_{2} \in \mathbb{R}^{\mathbb{R}}$ and put $F=\left\{f_{1}, f_{2}\right\}$. It is enough to find $g \in \mathbb{R}^{\mathbb{R}}$ such that $f_{1}+g, f_{2}+g \in \mathcal{F}$. But $f_{1}-f_{2} \in \mathbb{R}^{\mathbb{R}}=\mathcal{F}-\mathcal{F}$. So, there exist $h_{1}, h_{2} \in \mathcal{F}$ such that $f_{1}-f_{2}=h_{1}-h_{2}$. Put $g=h_{1}-f_{1}=h_{2}-f_{2}$. Then $f_{i}+g=f_{i}+\left(h_{i}-f_{i}\right)=h_{i} \in \mathcal{F}$ for $i=1,2$.

" $\Leftarrow$ " By Proposition 1.1(2) we have $\mathrm{A}(\mathcal{F}) \geq 2$. To see that $\mathrm{A}(\mathcal{F}) \leq 2$ let $h \in \mathbb{R}^{\mathbb{R}} \backslash(\mathcal{F}-\mathcal{F})$, take $F=\left\{\chi_{\emptyset}, h\right\}$ and choose an arbitrary $g \in \mathbb{R}^{\mathbb{R}}$. It is enough to show that $f+g \notin \mathcal{F}$ for some $f \in F$. But if $g=\chi_{\emptyset}+g \in \mathcal{F}$ and $h+g \in \mathcal{F}$, then $h \in \mathcal{F}-g \subset \mathcal{F}-\mathcal{F}$, contradicting the choice of $h$.

Now, let $X$ and $Y$ be topological spaces. In what follows we will consider the following classes of functions from $X$ into $Y$. (In fact, we will consider these classes mainly for $X=Y=\mathbb{R}$.)

$\mathrm{D}(X, Y)$ of Darboux functions $f: X \rightarrow Y$; i.e., such that $f[C]$ is connected in $Y$ for every connected subset $C$ of $X$.

$\operatorname{Conn}(X, Y)$ of connectivity functions $f: X \rightarrow Y$; i.e., such that the graph of $f$ restricted to $C$ (that is $f \cap[C \times Y]$ ) is connected in $X \times Y$ for every connected subset $C$ of $X$.

$\mathrm{AC}(X, Y)$ of almost continuous functions $f: X \rightarrow Y$; i.e., such that every open subset $U$ of $X \times Y$ containing the graph of $f$, there is a continuous function $g: X \rightarrow Y$ with $g \subset U$.

$\operatorname{Ext}(X, Y)$ of extendable functions $f: X \rightarrow Y$; i.e., such that there exists a connectivity function $g: X \times[0,1] \rightarrow Y$ with $f(x)=g(x, 0)$ for every $x \in X$.

PR of functions $f: \mathbb{R} \rightarrow \mathbb{R}$ with perfect road $(X=Y=\mathbb{R})$; i.e., such that for every $x \in \mathbb{R}$ there exists a perfect set $P \subseteq \mathbb{R}$ having $x$ as a bilateral limit point for which restriction $\left.f\right|_{P}$ of $f$ to $P$ is continuous at $x$.

$\mathrm{PC}(X, Y)$ of peripherally continuous functions $f: X \rightarrow Y$; i.e., such that for every $x \in X$ and any pair $U \subseteq X$ and $V \in Y$ of open neighborhoods of $x$ and $f(x)$, respectively, there exists an open neighborhood $W$ of $x$ with $\operatorname{cl}(W) \subseteq U$ and $f[\operatorname{bd}(W)] \subseteq V$, where $\operatorname{cl}(W)$ and $\operatorname{bd}(W)$ stand for the closure and the boundary of $W$, respectively.

We will write $\mathrm{D}$, Conn, $\mathrm{AC}$, Ext, and $\mathrm{PC}$ in place of $\mathrm{D}(X, Y), \operatorname{Conn}(X, Y)$, $\operatorname{AC}(X, Y), \operatorname{Ext}(X, Y)$, and $\mathrm{PC}(X, Y)$ if $X=Y=\mathbb{R}$. 
Note also, that function $f: \mathbb{R} \rightarrow \mathbb{R}$ is peripherally continuous $(f \in \mathrm{PC})$ if and only if for every $x \in \mathbb{R}$ there are sequences $a_{n} \nearrow x$ and $b_{n} \searrow x$ such that $\lim _{n \rightarrow \infty} f\left(a_{n}\right)=\lim _{n \rightarrow \infty} f\left(b_{n}\right)=f(x)$. In particular, if graph of $f$ is dense in $\mathbb{R}^{2}$, then $f$ is peripherally continuous.

For the classes of functions (from $\mathbb{R}$ into $\mathbb{R}$ ) defined above we have the following proper inclusions $\subset$, marked by arrows $\longrightarrow$. (See [2].)

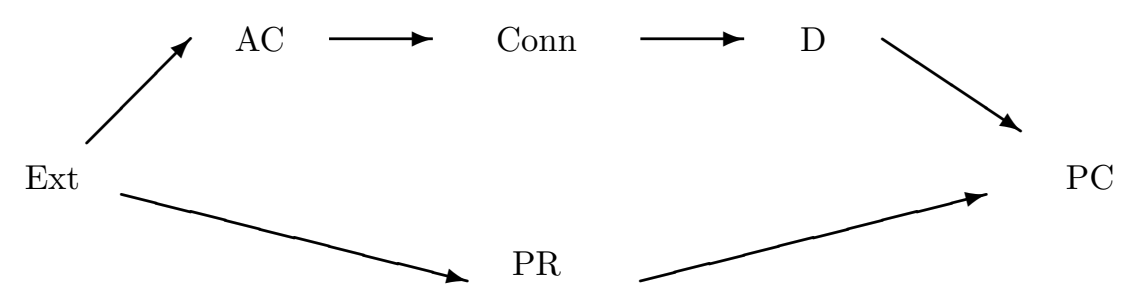

In what follows we will also use the following theorem due to Hagan [9].

Theorem 1.4 If $n \geq 2$, then $\operatorname{Conn}\left(\mathbb{R}^{n}, \mathbb{R}\right)=\mathrm{PC}\left(\mathbb{R}^{n}, \mathbb{R}\right)$.

The functions $\mathrm{A}$ and $\mathrm{M}$ for the classes $\mathrm{AC}$, Conn and D were studied in $[10,3,12]$. In particular, the following is known.

Theorem 1.5 $[12] \mathrm{M}(\mathrm{AC})=\mathrm{M}(\mathrm{Conn})=\mathrm{M}(\mathrm{D})=\operatorname{cf}(\mathfrak{c})$.

Theorem 1.6 $[3] \mathfrak{c}^{+} \leq \mathrm{A}(\mathrm{AC})=\mathrm{A}(\mathrm{Conn})=\mathrm{A}(\mathrm{D}) \leq 2^{\mathfrak{c}}, \operatorname{cf}(\mathrm{A}(\mathrm{D}))>\mathfrak{c}$ and it is pretty much all that can be shown in ZFC. More precisely, it is consistent with $Z F C$ that $\mathrm{A}(\mathrm{D})$ can be equal to any regular cardinal between $\mathfrak{c}^{+}$and $2^{\mathfrak{c}}$ and that it can be equal to $2^{\mathfrak{c}}$ independent of the cofinality of $2^{\mathfrak{c}}$.

The goal of this paper is to prove the following theorem.

Theorem 1.7 (1) $\mathrm{M}(\mathrm{PC})=\mathfrak{c}$.

(2) $\mathrm{M}($ Ext $)=\mathrm{M}(\mathrm{PR})=2$.

(3) $\mathrm{A}(\mathrm{PC})=2^{\mathfrak{c}}$.

(4) $\mathrm{A}(\mathrm{Ext})=\mathrm{A}(\mathrm{PR})=\mathfrak{c}^{+}$.

This will be proved in the next sections. Notice only that the equation $\mathrm{A}($ Ext $)=\mathfrak{c}^{+}$and Proposition 1.3 immediately imply the following corollary, which gives a positive answer to a question of Gibson [6]. (Compare also [13] and [14].)

Corollary 1.8 Every function $f: \mathbb{R} \rightarrow \mathbb{R}$ is the sum of two extendable functions. 


\section{Proof of Theorem 1.7(1), (2) and (3)}

Proof of $\mathrm{M}($ Ext $)=\mathrm{M}(\mathrm{PR})=2$. The inequalities $2 \leq \mathrm{M}($ Ext $) \leq \mathrm{M}(\mathrm{PR})$ follow from Proposition 1.2. To see that $\mathrm{M}(\mathrm{PR}) \leq 2$ take $F=\left\{\chi_{B}, \chi_{\mathbb{R} \backslash B}\right\}$ where $B \subset \mathbb{R}$ is a Bernstein set. Then for every $g \in \mathbb{R}^{\mathbb{R}} \backslash\left\{\chi_{\emptyset}\right\}$ we have $f \cdot g \neq \mathrm{PR}$ for some $f \in F$. To see it, take $x \in \mathbb{R}$ such that $g(x)=r \neq 0$. If $x \in B$, then $\chi_{B} \cdot g$ does not have a perfect road at $x$, since $\left(\chi_{B} \cdot g\right)(x)=r \neq 0$ and $\left(\chi_{B} \cdot g\right)^{-1}(0) \cap P \neq \emptyset$ for every perfect set $P \subseteq \mathbb{R}$. Similarly, $\chi_{\mathbb{R} \backslash B} \cdot g$ does not have a perfect road at $x$ if $x \in \mathbb{R} \backslash B$.

Proof of $\mathrm{M}(\mathrm{PC})=\mathfrak{c}$. The inequality $\mathrm{M}(\mathrm{PC}) \leq \mathfrak{c}$ follows from Proposition 1.2. So, it is enough to show that $\mathrm{M}(\mathrm{PC}) \geq \mathfrak{c}$.

Let $F \subseteq \mathbb{R}^{\mathbb{R}}$ be a family of cardinality less than or equal to $\kappa$ with $\omega \leq$ $\kappa<\mathfrak{c}$. We will find $g \in \mathbb{R}^{\mathbb{R}} \backslash \chi_{\emptyset}$ such that $f \cdot g \in$ PC for every $f \in F$. For $f: \mathbb{R} \rightarrow \mathbb{R}$ let $[f \neq 0]$ denote $\{x \in \mathbb{R}: f(x) \neq 0\}$ and let

$$
A_{f}=\left\{x \in \mathbb{R}: f(x) \neq 0 \&[f \neq 0] \text { is not bilaterally } \kappa^{+} \text {-dense at } x\right\},
$$

where set $S \subseteq \mathbb{R}$ is said to be bilaterally $\kappa^{+}$-dense at $x$ if for every $\varepsilon>0$ each of the sets $S \cap[x-\varepsilon, x]$ and $S \cap[x, x+\varepsilon]$ have cardinality at least $\kappa^{+}$. Note that $\left|A_{f}\right| \leq \kappa$ for every $f: \mathbb{R} \rightarrow \mathbb{R}$. This is the case, since for every $x \in A_{f}$ there exists a closed interval $J$ with non-empty interior such that $x \in J$ and $|[f \neq 0] \cap J| \leq \kappa$. Now, if $\mathcal{J}$ is the family of all maximal intervals $J$ with non-empty interior such that $|[f \neq 0] \cap J| \leq \kappa$, then $|\mathcal{J}| \leq \omega$, $A_{f} \subseteq \bigcup_{J \in \mathcal{J}}([f \neq 0] \cap J)$ and $\left|A_{f}\right| \leq\left|\bigcup_{J \in \mathcal{J}}([f \neq 0] \cap J)\right| \leq \kappa$.

Let $A=\bigcup_{f \in F} A_{f}$. Then $|A| \leq \kappa$. Notice that the set $[f \neq 0] \backslash A$ is bilaterally $\kappa^{+}$-dense at $x$ for every $f \in F$ and $x$ from $[f \neq 0] \backslash A$. To define $g$ let $\left\langle\left\langle f_{\alpha}, q_{\alpha}, I_{\alpha}\right\rangle: \alpha<\kappa\right\rangle$ be the sequence of all triples with $f_{\alpha} \in F, q_{\alpha} \in \mathbb{Q}$ and $I_{\alpha}$ be an open interval with rational end points. By induction define on $\alpha<\kappa$ a one-to-one sequence $\left\langle x_{\alpha}: \alpha<\kappa\right\rangle$ by choosing

(i) $x_{\alpha} \in\left[f_{\alpha} \neq 0\right] \cap\left(I_{\alpha} \backslash A\right) \backslash\left\{x_{\beta}: \beta<\alpha\right\}$ if the choice can be made, and

(ii) $x_{\alpha} \in\left(I_{\alpha} \backslash A\right) \backslash\left\{x_{\beta}: \beta<\alpha\right\}$ otherwise.

Now, for $x \in \mathbb{R}$ we put

$$
g(x)= \begin{cases}\frac{q_{\alpha}}{f_{\alpha}(x)} & \text { if there is } \alpha<\kappa \text { with } x=x_{\alpha} \text { and } f_{\alpha}(x) \neq 0 \\ 1 & \text { if there is } \alpha<\kappa \text { with } x=x_{\alpha} \text { and } f_{\alpha}(x)=0 \\ 0 & \text { otherwise }\end{cases}
$$

Then $g \neq \chi_{\emptyset}$ and for each $q \in \mathbb{Q}$ and $f \in F$ the set $(g \cdot f)^{-1}(q)$ is bilaterally dense at every $x$ from $[f \neq 0] \backslash A$. Moreover, $(g \cdot f)(x)=0$ outside of $[f \neq 0] \backslash A$ 
and $(g \cdot f)^{-1}(0)$ is bilaterally dense at every $x \in \mathbb{R}$. So, $g \cdot f \in$ PC for every $f \in F$.

To prove $\mathrm{A}(\mathrm{PC})=2^{\mathfrak{c}}$ we will use the following result.

Theorem 2.1 Let $A$ and $B$ be such that $|A|=\omega$ and $|B|=\mathfrak{c}$. Then there exists a family $\mathcal{C} \subseteq A^{B}$ of size $2^{\mathfrak{c}}$ such that for every one-to-one sequence $\left\langle g_{a} \in \mathcal{C}: a \in A\right\rangle$ there is $b \in B$ with $g_{a}(b)=a$ for every $a \in A$.

Proof. The theorem is proved in [4, Corollary 3.17, p. 77] for $A=\omega$ and $B=\mathfrak{c}$. The generalization is obvious.

From this we will conclude the following lemma.

Lemma 2.2 If $B \subseteq \mathbb{R}$ has cardinality $\mathfrak{c}$ and $H \subseteq \mathbb{Q}^{B}$ is such that $|H|<2^{\mathfrak{c}}$, then there is $g \in \mathbb{Q}^{B}$ such that $h \cap g \neq \emptyset$ for every $h \in H$.

Proof. Let $\mathcal{C}$ be as in Theorem 2.1 with $A=\mathbb{Q}$. For each $h \in H$ there only finitely many $g \in \mathcal{C}$ for which $h \cap g=\emptyset$, since any countable infinite subset of $\mathcal{C}$ can be enumerated as $\left\{g_{a} \in \mathcal{C}: a \in \mathbb{Q}\right\}$. So there is $g \in \mathcal{C}$ such that $h \cap g \neq \emptyset$ for every $h \in H$.

Proof of $\mathrm{A}(\mathrm{PC})=2^{\mathfrak{c}}$. By Proposition 1.1 to prove $\mathrm{A}(\mathrm{PC})=2^{\mathfrak{c}}$ it is enough to show that $\mathrm{A}(\mathrm{PC}) \geq 2^{\mathrm{c}}$. So, let $F \subseteq \mathbb{R}^{\mathbb{R}}$ be such that $|F|<2^{\mathrm{c}}$. We will find $g: \mathbb{R} \rightarrow \mathbb{R}$ such that $f+g \in$ PC for every $f \in F$. Let $\mathcal{G}$ be the family of all triples $\langle I, p, m\rangle$ where $I$ is a non-empty open interval with rational end points, $p \in \mathbb{Q}$ and $m<\omega$. For each $\langle I, p, m\rangle \in \mathcal{G}$ define a set $B_{\langle I, p, m\rangle} \subseteq I$ of size $\mathfrak{c}$ such that $B_{\langle I, p, m\rangle} \cap B_{\langle J, q, n\rangle}=\emptyset$ for any distinct $\langle I, p, m\rangle$ and $\langle J, q, n\rangle$ from $\mathcal{G}$.

Next, fix $\langle I, p, m\rangle \in \mathcal{G}$ and for each $f \in F$ choose $h_{\langle I, p, m\rangle}^{f}: B_{\langle I, p, m\rangle} \rightarrow$ $\mathbb{Q}$ such that $\left|p-\left(f(x)+h_{\langle I, p, m\rangle}^{f}(x)\right)\right|<\frac{1}{m}$ for every $x \in B_{\langle I, p, m\rangle}$. Then, by Lemma 2.2 used with a set $H_{\langle I, p, m\rangle}=\left\{h_{\langle I, p, m\rangle}^{f}: f \in F\right\}$, there exists $g_{\langle I, p, m\rangle}: B_{\langle I, p, m\rangle} \rightarrow \mathbb{Q}$ such that

$$
\forall f \in F \exists x \in B_{\langle I, p, m\rangle} h_{\langle I, p, m\rangle}^{f}(x)=g_{\langle I, p, m\rangle}(x) .
$$

In particular, if $g: \mathbb{R} \rightarrow \mathbb{Q}$ is a common extension of all functions $g_{\langle I, p, m\rangle}$, then for every $\langle I, p, m\rangle \in \mathcal{G}$ and every $f \in F$ there exists $x \in B_{\langle I, p, m\rangle} \subseteq I$ such that

$$
|p-(f(x)+g(x))|<\frac{1}{m} .
$$

So, for every $f \in F$ the graph of $f+g$ is dense in $\mathbb{R}^{2}$. Thus, $f+g \in$ PC. 


\section{Proof of Theorem 1.7(4): $\mathrm{A}(\mathrm{Ext})=\mathrm{A}(\mathrm{PR})=\mathfrak{c}^{+}$}

By Proposition 1.1 we have $\mathrm{A}(\mathrm{Ext}) \leq \mathrm{A}(\mathrm{PR})$. Thus, it is enough to prove two inequalities: $\mathrm{A}(\mathrm{PR}) \leq \mathfrak{c}^{+}$and $\mathrm{A}(\mathrm{Ext}) \geq \mathfrak{c}^{+}$.

First we will prove $A(P R) \leq \mathfrak{c}^{+}$. For this we need the following lemma.

Lemma 3.1 There is a family $F \subseteq \mathbb{R}^{\mathbb{R}}$ of size $\mathfrak{c}^{+}$such that for every distinct $f, h \in F$, every perfect set $P$ and every $n<\omega$ there exists an $x \in P$ with $|f(x)-h(x)| \geq n$.

Proof. The family $F=\left\{f_{\xi}: \xi<\mathfrak{c}^{+}\right\}$is constructed by induction using a standard diagonal argument. If for some $\xi<\mathfrak{c}^{+}$the functions $\left\{f_{\zeta}: \zeta<\xi\right\}$ are already constructed, we construct $f_{\xi}$ as follows. Let $\left\langle\left\langle P_{\alpha}, h_{\alpha}, n_{\alpha}\right\rangle: \alpha<\mathfrak{c}\right\rangle$ be an enumeration of all triples $\langle P, h, n\rangle$ where $P \subseteq \mathbb{R}$ is perfect, $h=f_{\zeta}$ for some $\zeta<\xi$ and $n<\omega$. By induction on $\alpha<\mathfrak{c}$ choose $x_{\alpha} \in P_{\alpha} \backslash\left\{x_{\beta}: \beta<\alpha\right\}$ and define $f_{\xi}\left(x_{\alpha}\right)=h_{\alpha}\left(x_{\alpha}\right)+n_{\alpha}$. Then any extension of $f_{\xi}$ to $\mathbb{R}$ will have the desired properties.

Proof of $\mathrm{A}(\mathrm{PR}) \leq \mathfrak{c}^{+}$. Now let $F$ be a family from Lemma 3.1. We will show that for every $g: \mathbb{R} \rightarrow \mathbb{R}$ there exists $f \in F$ such that $f+g \notin \mathrm{PR}$. By way of contradiction assume that there exists a function $g: \mathbb{R} \rightarrow \mathbb{R}$ such that $f+g \in \mathrm{PR}$ for every $f \in F$. Then, for every $f \in F$ there exists a perfect set $P_{f}$ such that 0 is a bilateral limit point of $P_{f}$ and $\left.(f+g)\right|_{P_{f}}$ is continuous at 0 . Since there are $\mathfrak{c}^{+}$-many functions in $F$ and only $\mathfrak{c}$-many perfect sets, there are distinct $f, h \in F$ with $P_{f}=P_{h}$. Then the function $\left.((f+g)-(h+g))\right|_{P_{f}}=\left.(f-h)\right|_{P_{f}}$ is continuous at 0 contradicting the choice of the family $F$.

The proof of $\mathrm{A}(\mathrm{Ext}) \geq \mathfrak{c}^{+}$is based on the following facts.

Lemma 3.2 For every meager subset $M$ of $\mathbb{R}$ there exists a family $\left\{h_{\xi} \in\right.$ $\left.\mathbb{R}^{\mathbb{R}}: \xi<\mathfrak{c}\right\}$ of increasing homeomorphisms such that $h_{\zeta}[M] \cap h_{\xi}[M]=\emptyset$ for every $\zeta<\xi<\mathfrak{c}$.

Proof. Let $\left\{D_{\zeta}: \zeta<\mathfrak{c}\right\}$ be a family of pairwise disjoint $\mathfrak{c}$-dense, meager $F_{\sigma^{-}}$ sets. Then by $[8$, Lemma 4$]$ there are homeomorphisms $\left\{h_{\zeta}: \mathbb{R} \rightarrow \mathbb{R}: \zeta<\mathfrak{c}\right\}$ such that $h_{\zeta}[M] \subset D_{\zeta}$.

For $f \in \mathcal{F} \subset \mathbb{R}^{\mathbb{R}}$ we say that a set $G \subseteq \mathbb{R}$ is $f$-negligible for the class $\mathcal{F}$ provided $g \in \mathcal{F}$ for every $g: \mathbb{R} \rightarrow \mathbb{R}$ such that $\left.g\right|_{\mathbb{R} \backslash G}=\left.f\right|_{\mathbb{R} \backslash G}$. Thus, $G \subseteq \mathbb{R}$ is $f$-negligible for $\mathcal{F}$ if we can modify $f$ arbitrarily on $G$ remaining in the class $\mathcal{F}$.

Theorem 3.3 There exists a connectivity function $f: \mathbb{R}^{2} \rightarrow \mathbb{R}$ with graph dense in $\mathbb{R}^{3}$ such that some dense $G_{\delta}$ subset $G$ of $\mathbb{R}^{2}$ is $f$-negligible for the class $\operatorname{Conn}\left(\mathbb{R}^{2}, \mathbb{R}\right)$. 
The proof of this theorem will be postponed to the end of this section.

Corollary 3.4 There exists an extendable function $\hat{f}: \mathbb{R} \rightarrow \mathbb{R}$ with graph dense in $\mathbb{R}^{2}$ such that some dense $G_{\delta}$ subset $\hat{G}$ of $\mathbb{R}$ is $\hat{f}$-negligible for the class Ext.

Proof. Let $f: \mathbb{R}^{2} \rightarrow \mathbb{R}$ and $G$ be as in Theorem 3.3. Then there exists $y \in \mathbb{R}$ with $G^{y}=\{x:\langle x, y\rangle \in G\}$ being a dense $G_{\delta}$ subset of $\mathbb{R}$. Clearly the set $\hat{G}=G^{y}$ and the function $\hat{f}: \mathbb{R} \rightarrow \mathbb{R}$ defined by $\hat{f}(x)=f(x, y)$ for every $x \in \mathbb{R}$ satisfy the requirements.

The existence of a function as in Corollary 3.4 was first announced by H. Rosen at the 10th Auburn Miniconference in Real Analysis, April 1995. However, the construction presented at that time had a gap. This gap was removed later, as described in [14].

The construction presented in this paper is an independently discovered repair of the original Rosen's gap. It is also more general than that of [14], since [14] does not contain any example similar to that of Theorem 3.3.

Next, we will show how Lemma 3.2 and Corollary 3.4 imply A(Ext) $\geq \mathfrak{c}^{+}$. The argument is a modification of the proof of Corollary 1.8. (Compare also [11] and [14].)

Proof of $\mathrm{A}\left(\right.$ Ext) $\geq \mathfrak{c}^{+}$. Let $F=\left\{f_{\xi} \in \mathbb{R}^{\mathbb{R}}: \xi<\mathfrak{c}\right\}$. We will find $g: \mathbb{R} \rightarrow \mathbb{R}$ such that $f_{\xi}+g \in$ Ext for every $\xi<\mathfrak{c}$. So, let $\hat{f}: \mathbb{R} \rightarrow \mathbb{R}$ and $\hat{G} \subseteq \mathbb{R}$ be as in Corollary 3.4. Put $M=\mathbb{R} \backslash \hat{G}$ and take $\left\{h_{\xi} \in \mathbb{R}^{\mathbb{R}}: \xi<\mathfrak{c}\right\}$ as in Lemma 3.2. For $\xi<\mathfrak{c}$ define $g$ on $h_{\xi}[M]$ to be $\left.\left(\hat{f} \circ h_{\xi}^{-1}-f_{\xi}\right)\right|_{h_{\xi}[M]}$ and extend it to $\mathbb{R}$ arbitrarily. To see that $f_{\xi}+g \in$ Ext note that $f_{\xi}+g=\hat{f} \circ h_{\xi}^{-1}$ on $h_{\xi}[M]$. But the set $\mathbb{R} \backslash h_{\xi}[M]=h_{\xi}[\hat{G}]$ is $\left(\hat{f} \circ h_{\xi}^{-1}\right)$-negligible for the class Ext. (See [11] for an easy proof.) So, each $f_{\xi}+g$ is extendable.

Proof of Theorem 3.3. We will construct a peripherally continuous function $f: \mathbb{R}^{2} \rightarrow \mathbb{R}$ with dense $G_{\delta}$ subset $G$ of $\mathbb{R}^{2}$ which is $f$-negligible for the class $\operatorname{PC}\left(\mathbb{R}^{2}, \mathbb{R}\right)$. It is enough since, by Theorem 1.4 , Conn $\left(\mathbb{R}^{2}, \mathbb{R}\right)=\operatorname{PC}\left(\mathbb{R}^{2}, \mathbb{R}\right)$. The construction is a modification of that from [7], where a similar example of a function from $[0,1] \times[0,1]$ onto $[0,1]$ was constructed. (Compare also [1].) The additional difficulty in our construction is to make sure that some sequences of points in the range of $f(=\mathbb{R})$ have cluster points, which is obvious for all sequences in $[0,1]$. Also, our basic construction step will be based on a triangle, while the construction in [7] was based on a square. Triangles work better, since for three arbitrary non-collinear points in $\mathbb{R}^{3}$ there is precisely one plane passing through them, while it is certainly false for four points.

Basic Idea: We will construct, by induction on $n<\omega$, a sequence $\left\langle S_{n}: n<\omega\right\rangle$ of triangular "grids" formed with equilateral triangles of side length $1 / 2^{k_{n}}$, as 


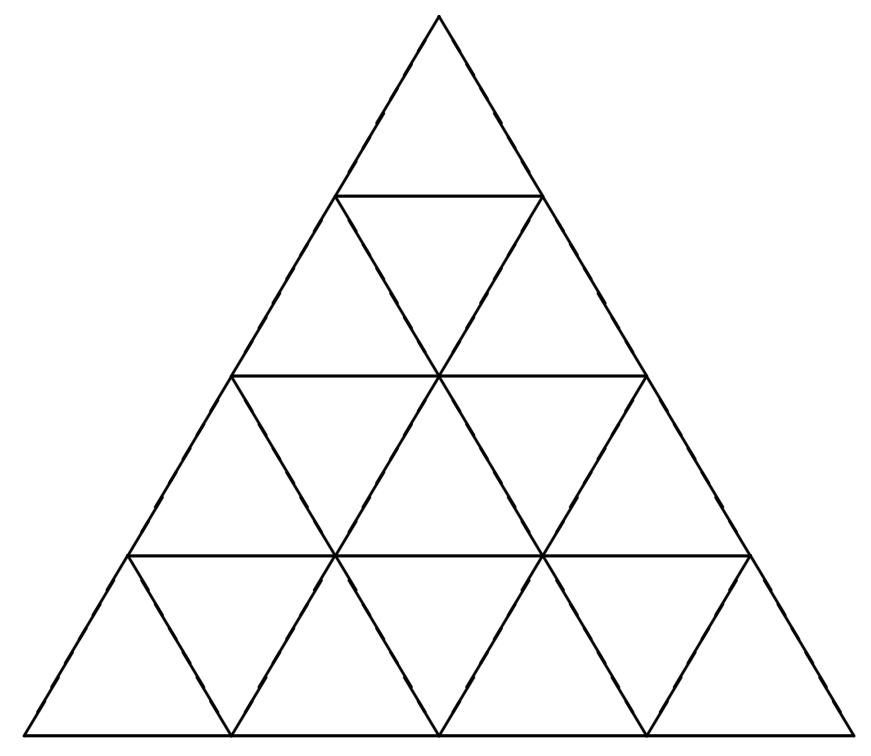

Figure 1: Grid $S_{n}$

in Figure 1. The grid $S_{n}$ will be identified with the points on the edges of triangles forming it and we will be assuming that $S_{n} \subseteq S_{n+1}$ for all $n<\omega$. With each grid $S_{n}$ we will associate a continuous function $f_{n}: S_{n} \rightarrow \mathbb{R}$ which is linear on each side of a triangle from $S_{n}$. Moreover, each $f_{n+1}$ will be an extension of $f_{n}$. Function $f$ will be defined as an extension of $\bigcup_{n<\omega} f_{n}$.

Terminology: In what follows a triangle will be identified with the set of points of its interior or its boundary.

For a grid $S$ we say that a triangle $T$ is from $S$ if the interior of $T$ is equal to a component of $\mathbb{R}^{2} \backslash S$.

For an equilateral triangle $T$, its basic partition will be its division into seven equilateral triangles, as in Figure 2. The central triangle $\hat{T}$ of Figure 2 will be referred as the middle quarter of $T$. Thus, $\hat{T} \cap \operatorname{bd}(T)=\emptyset$ and the length of each side of $\hat{T}$ is equal to $1 / 4$ of the length of a side of $T$.

If a function $F$ is defined on the three vertices of a triangle $T$, its basic extension is defined as the unique function $\hat{F}: T \rightarrow \mathbb{R}$ extending $F$ whose graph is a subset of a plane. Notice, that $\hat{F}$ is linear on each side of the triangle $T$ and that $\hat{F}$ extends $F$ even if the function $F$ has already been defined on some side of $T$ as long as $F$ is linear on this side.

Inductive Construction: We will define inductively three increasing sequences $\left\langle S_{n}: n<\omega\right\rangle$ of triangular grids as in Figure $1,\left\langle f_{n} \in \mathbb{R}^{S_{n}}: n<\omega\right\rangle$ of 


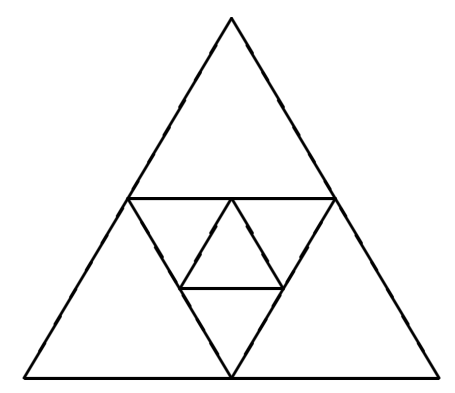

Figure 2: Basic partition

continuous functions and $\left\langle k_{n}<\omega: n<\omega\right\rangle$ of natural numbers such that the following inductive conditions are satisfied for every $n<\omega$.

(i) $f_{n}: S_{n} \rightarrow\left[-2^{n}, 2^{n}\right]$ and is linear on each side of a triangle $T$ from $S_{n}$.

(ii) The side length of each triangle from $S_{n}$ is equal to $1 / 2^{k_{n}}$.

(iii) The variation of $f_{n}$ on each triangle from $S_{n}$ is $\leq 1 / 2^{n}$.

(iv) If $n>0$, then for every triangle $T$ from $S_{n-1}$ and every dyadic number $i / 2^{n} \in\left[-2^{n}, 2^{n}\right]$ with $i \in \mathbb{Z}\left(-4^{n} \leq i \leq 4^{n}\right)$ there is a triangle $T_{i} \subseteq \hat{T}$ such that $\mathrm{bd}\left(T_{i}\right) \subseteq S_{n}$ and $f_{n}(x)=i / 2^{n}$ for every $x \in \operatorname{bd}\left(T_{i}\right)$.

(v) If $n>0, T$ is a triangle from $S_{n-1}$ and $T^{\prime}$ is a triangle from $S_{n}$ such that $T^{\prime} \subseteq T$ and $T^{\prime} \nsubseteq \hat{T}$, then $f_{n}\left[\operatorname{bd}\left(T^{\prime}\right)\right] \subseteq[-M, M]$, where $M=$ $\max \left\{\left|f_{n-1}(x)\right|: x \in \operatorname{bd}(T)\right\}$.

To start the induction, take $k_{0}=0$, define grid $S_{0}$ as in Figure 1 with all sides of length $1=1 / 2^{0}$ and choose $f_{0}: S_{0} \rightarrow \mathbb{R}$ as constantly equal 0 . It is easy to see that the conditions (i) $-(\mathrm{v})$ are satisfied with such a choice.

Next, assume that for some $n>0$ we already have $S_{n-1}, f_{n-1}$ and $k_{n-1}$ satisfying (i)-(v). We will define $S_{n}$, find $k_{n}$ and extend $f_{n-1}$ to $f_{n}: S_{n} \rightarrow \mathbb{R}$ such that (i)-(v) will still hold. Put $F_{n}=f_{n-1}$.

Step 1. Let $T$ be a triangle from $S_{n-1}$ and extend $F_{n}$ into each vertex of its middle quarter $\hat{T}$ by assigning it the value 0 . Notice, that $F_{n}$ is defined on all vertices of the basic partition of $T$.

Partition $\hat{T}$ into a grid $S$ such that the size of each triangle from $S$ is equal $1 / 2^{\hat{k}_{n}}$. The number $\hat{k}_{n}<\omega$ is chosen as a minimal number such that there are $2 \cdot 8^{n}+1$ disjoint triangles $\left\{T_{i}: i \in \mathbb{Z},-4^{n} \leq i \leq 4^{n}\right\}$ from $S$ none of 


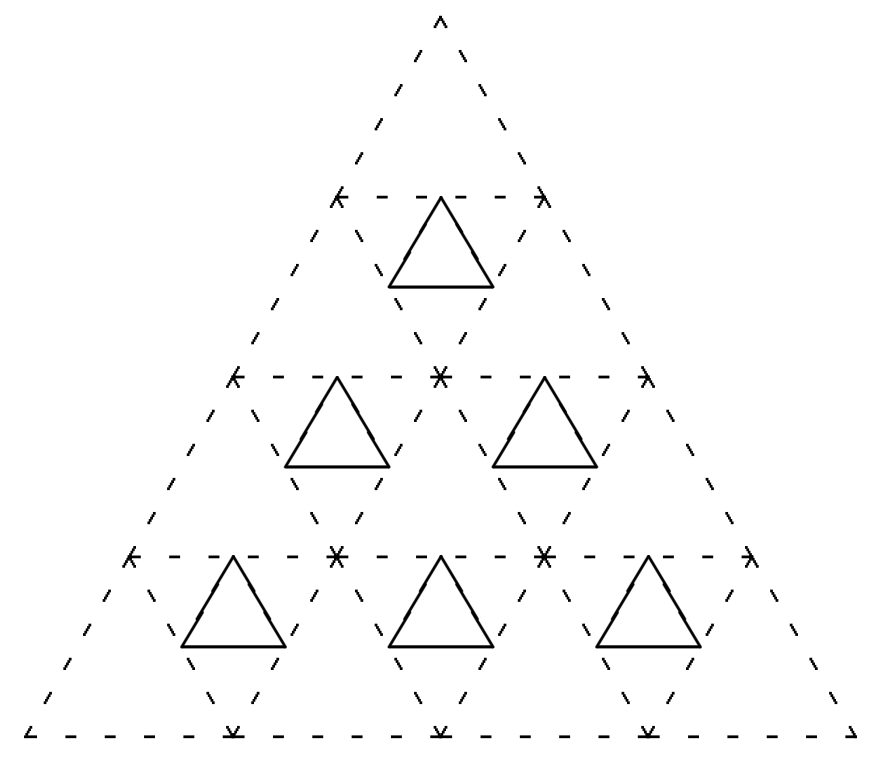

Figure 3: Some triangles of the grid of $\hat{T}$

which intersects the boundary of $\hat{T}$. (See Figure 3.) Notice that the value of $\hat{k}_{n}$ does not depend on $T$. On the vertices of each triangle $T_{i}$ define $F_{n}$ to be equal $i / 2^{n}$. On the remaining undefined vertices of $S$ define $F_{n}$ to be equal 0 . Notice that $F_{n}$ is defined on all vertices of each triangle defined so far.

Step 2. Extend $F_{n}$ into $\mathbb{R}^{2}$, by defining it on every triangle $T$ constructed so far as the basic extension of $\left.F_{n}\right|_{\mathrm{bd}(T)}$.

Notice that if we extend grid $S_{n-1}$ to the grid $\hat{S}_{n}$ with side length of each triangle from $\hat{S}_{n}$ equal $1 / 2^{\hat{k}_{n}}$ and put $\hat{f}_{n}=\left.F_{n}\right|_{\hat{S}_{n}}$, then the triple $\left\langle\hat{S}_{n}, \hat{f}_{n}, \hat{k}_{n}\right\rangle$ satisfies conditions (i), (ii), (iv) and (v).

Step 3 . We have to modify $\hat{S}_{n}, \hat{f}_{n}$ and $\hat{k}_{n}$ to also get condition (iii), while keeping the other properties. First notice that for every triangle $T$ from $\hat{S}_{n}$ and any interval $J$ inside $T$ the slope of $F_{n}$ on $J$ does not exceed the number $\frac{\text { length of the range of } F_{n}}{\text { length of a side of } T} \leq \frac{22^{n}}{2^{-\hat{k}_{n}}}=2^{n \hat{k}_{n}+1}$. So, let $k_{n} \geq n \hat{k}_{n}+n+1$, in which case

$$
\frac{1}{2^{k_{n}}} 2^{n \hat{k}_{n}+1} \leq \frac{1}{2^{n}}
$$

let $S_{n}$ be a refinement of the grid $\hat{S}_{n}$ with triangles with side size $1 / 2^{k_{n}}$ and put $f_{n}=\left.F_{n}\right|_{S_{n}}$. It is easy to see that this gives us (iii) while preserving the other conditions. This finishes the inductive construction. 
Let $S=\bigcup_{n<\omega} S_{n}$ and define $f$ on $S$ by $f=\bigcup_{n<\omega} f_{n}$. To extend it to $\mathbb{R}^{2} \backslash S$ notice that

$(\star)$ for every $x \in \mathbb{R}^{2} \backslash S$ there exists a number $f(x) \in \mathbb{R}$ and a sequence $\left\langle T_{k}: k<\omega\right\rangle$ of triangles with $x$ being an interior point of each $T_{k}$ such that $\lim _{k \rightarrow \infty} \operatorname{diam}\left(T_{k}\right) \rightarrow 0$ and

$$
f(x)=\lim _{k \rightarrow \infty} \min f\left[\operatorname{bd}\left(T_{k}\right)\right]=\lim _{k \rightarrow \infty} \max f\left[\operatorname{bd}\left(T_{k}\right)\right] .
$$

The proof of $(\star)$ will finish the construction of $f$.

To see $(\star)$ fix $x \in \mathbb{R}^{2} \backslash S$ and let $T_{n}^{0}$ be the triangle from $S_{n}$ such that $x$ belongs to the interior of $T_{n}^{0}$. Let $N=\left\{n<\omega: n>0 \& T_{n}^{0} \subseteq \hat{T}_{n-1}^{0}\right\}$. There are two cases to consider.

Case 1. The set $N$ is infinite. Then, let $\left\langle n_{k}: k\langle\omega\rangle\right.$ be a one-to-one enumeration of $N$ and define $T_{k}=\hat{T}_{n_{k}-1}^{0}$. It is easy to see that this sequence satisfies $(\star)$ with $f(x)=0$.

Case 2. The set $N$ is finite. Let $m<\omega$ be such that $T_{n}^{0} \nsubseteq \hat{T}_{n-1}^{0}$ for every $n \geq m$ and let $M=\max \left\{\left|f_{m-1}(x)\right|: x \in \operatorname{bd}\left(T_{m-1}^{0}\right)\right\}$. Then, by condition (v), $f_{n}\left[\operatorname{bd}\left(T_{n}^{0}\right)\right] \subseteq[-M, M]$ for every $n \geq m$. So, there exists an increasing sequence $\left\langle n_{k} \geq m: k<\omega\right\rangle$ such that $L=\lim _{k \rightarrow \infty} \max f\left[\operatorname{bd}\left(T_{n_{k}}^{0}\right)\right]$ exists. It is easy to see that the sequence $\left\langle T_{k}\right\rangle=\left\langle T_{n_{k}}^{0}\right\rangle$ satisfies $(\star)$ with $f(x)=L$, since the variation of $f$ on $\operatorname{bd}\left(T_{n_{k}}^{0}\right)$ tends to 0 as $k \rightarrow \infty$.

This finishes the construction of function $f$. It remains to show that $f$ has the desired properties.

Clearly $(\star)$ implies that $f$ is peripherally continuous at every point $x \in$ $\mathbb{R}^{2} \backslash S$. To see that $f$ is peripherally continuous on $S$ take $x \in S$. Then, there exists $k<\omega$ such that $x \in S_{n}$ for every $n \geq k$. For any such $n$ let $\mathcal{T}_{n}$ be the set of all triangles from $S_{n}$ to which $x$ belongs. Notice that $\mathcal{T}_{n}$ has at most six elements and that $x$ belongs to the interior of the polygon $P_{n}=\bigcup \mathcal{T}_{n}$. Hence, the variation on the boundary of $P_{n}$ is at most $6 / 2^{n}$ and the diameter of $P_{n}$ is at most $1 / 2^{n-1}$. So, the sequence $\left\langle P_{n}\right\rangle$ guarantees that $f$ is peripherally continuous at $x$.

To finish the proof it is enough to find a dense $G_{\delta}$ set $G$ which is $f$-negligible for $\operatorname{PC}\left(\mathbb{R}^{2}, \mathbb{R}\right)$. For any dyadic number $d$ and any $k \in \omega$ let $\mathcal{F}_{d}^{k}$ denote the family of all triangles $T$ for which there exists $n \geq k$ such that $T$ is from $S_{n}$ and $f_{n}(x)=d$ for every $x \in \operatorname{bd}(T)$. Let $G_{d}^{k}$ be the union of the interiors of all triangles $T \in \mathcal{F}_{d}^{k}$. Then, by condition (iv), each set $G_{d}^{k}$ is open and dense. Therefore, $G=\bigcap\left\{G_{d}^{k}: k \in \omega \& d\right.$ is dyadic $\}$ is a dense $G_{\delta}$ set. It is easy to see, that $f$ is peripherally continuous if we redefine it on the set $G$ in an arbitrary way.

This finishes the proof of Theorem 3.3. 


\section{Compositions of Lebesgue Measurable Functions}

We can consider similar problems for compositions of functions. For example, we know that every function is a composition of Lebesgue measurable functions [15]. (See also Problem 6378, American Mathematical Monthly, 90, 573.) It is easy to make every function in $\mathbb{R}^{\mathbb{R}}$ measurable (in a sense of definition of A) using composition with just one function. We simply take a composition with a constant function. So we need to define cardinal invariants in a different way.

The next definition will represent one of the ways the problem can be approached. Instead of "forcing the family $H$ to be in $\mathcal{F}$ " we will try to recover all elements of $H$ with one "coding" function $\hat{f} \in \mathcal{F}$ and the class $\mathcal{F}$ of all codes. This leads to the following definitions.

$C_{r}(\mathcal{F})=\min \left\{|H|: H \subseteq \mathbb{R}^{\mathbb{R}} \& \neg \exists \hat{f} \in \mathcal{F} \forall h \in H \exists f \in \mathcal{F} f \circ \hat{f}=h\right\} \cup\left\{\left(2^{\mathfrak{c}}\right)^{+}\right\}$

and

$C_{l}(\mathcal{F})=\min \left\{|H|: H \subseteq \mathbb{R}^{\mathbb{R}} \& \neg \exists \hat{f} \in \mathcal{F} \forall h \in H \exists f \in \mathcal{F} \hat{f} \circ f=h\right\} \cup\left\{\left(2^{\mathfrak{c}}\right)^{+}\right\}$.

Let $\mathcal{L}$ be the family of all Lebesgue measurable functions from $\mathbb{R}$ into $\mathbb{R}$.

Theorem 4.1 $C_{r}(\mathcal{L})=\left(2^{\mathfrak{c}}\right)^{+}$and $C_{l}(\mathcal{L})=\mathfrak{c}^{+}$.

Proof. To see $C_{r}(\mathcal{L}) \geq\left(2^{\mathfrak{c}}\right)^{+}$we will show that the family $H=\mathbb{R}^{\mathbb{R}}$ of all functions can be "coded" by one function $\hat{f} \in \mathcal{L}$. Simply, let $\hat{f}$ be a Borel isomorphism from $\mathbb{R}$ onto the Cantor ternary set $C$. For any function $h \in \mathbb{R}^{\mathbb{R}}$ we define $f_{h} \in \mathcal{L}$ by putting $f_{h} \equiv 0$ on the complement of $C$ and $f_{h}=h \circ \hat{f}^{-1}$ on $C$. Then $h=f_{h} \circ \hat{f}$.

To see that $C_{l}(\mathcal{L}) \geq \mathfrak{c}^{+}$let $H=\left\{h_{\xi}: \xi<\mathfrak{c}\right\} \subseteq \mathbb{R}^{\mathbb{R}}$ and let $\left\{C_{\xi}: \xi<\mathfrak{c}\right\}$ be a partition of the Cantor ternary set $C$ into perfect sets. Then for every $\xi<\mathfrak{c}$ take a Borel isomorphism $f_{\xi}: \mathbb{R} \rightarrow C_{\xi}$ and define $\hat{f}(x)=\left(h_{\xi} \circ f_{\xi}^{-1}\right)(x)$ for $x \in C_{\xi}$ and $\hat{f}(x)=0$ otherwise. It is easy to see that $\hat{f} \in \mathcal{L}$ and $\hat{f} \circ f_{\xi}=h_{\xi}$ for every $\xi<\mathfrak{c}$.

To prove $C_{l}(\mathcal{L}) \leq \mathfrak{c}^{+}$take $\left\{h_{\xi}: \xi<\mathfrak{c}^{+}\right\}$from Lemma 3.1. Assume that there exists a sequence $\left\{f_{\xi}: \xi<\mathfrak{c}^{+}\right\}$of measurable functions and a measurable function $\hat{f}$ such that $h_{\xi}=\hat{f} \circ f_{\xi}$ for every $\xi<\mathfrak{c}^{+}$. For each $\xi<\mathfrak{c}^{+}$let $P_{\xi}$ be a perfect set such that $f_{\xi} \mid P_{\xi}$ is continuous. Then, by a cardinality argument, there are $\zeta<\xi<\mathfrak{c}^{+}$such that $P_{\zeta}=P_{\xi}$ and $f_{\zeta}\left|P_{\zeta}=f_{\xi}\right| P_{\xi}$. So, $h_{\zeta}\left|P_{\zeta}=h_{\xi}\right| P_{\xi}$. Contradiction. 


\section{References}

[1] J. B. Brown, Totally discontinuous connectivity functions, Coll. Math., 23 (1971), 53-60.

[2] J. B. Brown, P. Humke, and M. Laczkovich, Measurable Darboux functions, Proc. Amer. Math. Soc., 102 (1988), 603-609.

[3] K. Ciesielski and A. W. Miller, Cardinal invariants concerning functions, whose sum is almost continuous, Real Analysis Exch., 20 (1994-95), 657672 .

[4] W. W. Comfort and S. Negrepontis, The Theory of Ultrafilters, Springer Verlag, New York, 1974.

[5] H. Fast, Une remarque sur la propriété de Weierstrass, Colloquium Mathematicum, 7 (1959), 75-77.

[6] R. G. Gibson, A property of Borel measurable functions and extendable functions, Real Analysis Exch., 13 (1987-88), 11-15.

[7] R. G. Gibson and F. Roush, The restrictions of a connectivity function are nice but not that nice, Real Anal. Exchange, 12 (1986-87), 372-376.

[8] W. J. Gorman III, The homeomorphic transformations of c-sets into dsets, Proc. Amer. Math. Soc., 17 (1966), 826-830.

[9] M. Hagan, Equivalence of connectivity maps and peripherally continuous transformations, Proc. Amer. Math. Soc., 17 (1966), 175-177.

[10] T. Natkaniec, Almost Continuity,Real Analysis Exch., 17, (1991-92), 462520 .

[11] T. Natkaniec, Extendability and almost continuity, Real Analysis Exch., 21 (1995-96), 349-355.

[12] T. Natkaniec and I. Recław, Cardinal invariants concerning functions whose product is almost continuous, Real Analysis Exch., 20 (1994-95), 281-285.

[13] H. Rosen, Limits and sums of extendable connectivity functions, Real Analysis Exch., 20 (1994-95), 183-191.

[14] H. Rosen, Every Real Function is the Sum of Two Extendable Connectivity Functions, Real Analysis Exch., 21 (1995-96), 299-303.

[15] S. Ruziewicz, Sur une propriété des functions arbitraires d'une variable réelle, Mathematica, 9 (1935), 83-85. 